\title{
Simultaneous Determination of Hydroxyanthraquinones in Rhubarb and Experimental Animal Bodies by High-Performance Liquid Chromatography
}

\author{
Mingyu Ding, ${ }^{* \dagger}$ Shuaiwu MA,** and Delin LIU** \\ * Department of Chemistry, Tsinghua University, Beijing 100084, China \\ **Institute of Basic Theory of TCM, China Academy of Traditional Chinese Medicine, Beijing 100700, China
}

\begin{abstract}
A simple and reliable high-performance liquid chromatographic (HPLC) method was developed for the simultaneous determination of five hydroxyanthraquinones (aloe-emodin, rhein, emodin, chrysophanol, and physcion) in Rhubarb and experimental animal bodies. A Zorbax SB-C18 column $(250 \mathrm{~mm} \times 4.6 \mathrm{~mm}$ i.d., $5 \mu \mathrm{m})$ and a methanol- $0.5 \%$ acetic acid $(85: 15, v / v)$ mobile phase were used for the separation. The detection wavelength of a diode array detector (DAD) was set at $254 \mathrm{~nm}$. Regression equations revealed a linear relationship $\left(R^{2}>0.9996\right)$ between the mass of hydroxyanthraquinones injected and the peak areas detected by DAD. The detection limits $(S / N=3)$ ranged from $0.35 \mathrm{ng}$ to $3.13 \mathrm{ng}$, and the recoveries ranged from $83 \%$ to $103 \%$ for different hydroxyanthraquinones. This method is simple, sensitive and suitable for the analysis of hydroxyanthraquinones in medicinal materials and pharmacological experiment samples.
\end{abstract}

(Received May 12, 2003; Accepted June 9, 2003)

Rhubarb is the dried rhizome of Rheum palmatum L., Rheum tanguticum Maxim. ex Balf., or R. Rheum officinale Baill. It is an important traditional Chinese medicinal material. It has shown a very strong antibacterial action, and is usually used for the treatment of bacterial dysentery. The main active compounds in Rhubarb are five hydroxyanthraquinones (chrysophanol, emodin, physcion, aloe-emodin and rhein) and their glucosides. The matrix chemical structures of these five hydroxyanthraquinones are the same (Fig. 1). Pharmacological experiments revealed that all of these hydroxyanthraquinones are effective on dysentery bacillus, typhoid bacillus and cholera bacillus. Among these hydroxyanthraquinones, aloe-emodin is the most potent, and rhein has been found to have a strong antibacterial action on Bacteroides fragilis.

Many of high-performance liquid chromatographic (HPLC) methods have been employed to analyze the five hydroxyanthraquinones, ${ }^{1-8}$ but some of them require a complicated mobile phase; for example, needs the addition of some buffer or other solvents into the methanol-base mobile phase, and most of them must use gradient elution methods. In this work, an isocratic elution HPLC method with the most simple mobile phase (methanol solution including acetic acid) and the most common stationary phase (ODS) was developed for the simultaneous separation of the five hydroxyanthraquinones in rhubarb medicinal material and in plasma, urine and cerebrospinal fluid (CSF) of experimental animals being fed the drug. This method shows a high sensitivity and a good resolution, and is suitable for the determination of Chinese medicinal materials and relative pharmacological samples.

$\dagger$ To whom correspondence should be addressed.

E-mail: dingmy@chem.tsinghua.edu.cn

\section{Experimental}

\section{Apparatus and chemicals}

The HP1100 HPLC system (Hewlett-Packard, USA) consists of a quaternary pump, a vacuum degasser, a thermostated column compartment, a diode array detection (DAD), a $20 \mu \mathrm{l}$ manual injector and an HP Chemstation.

All of the chemicals used in this study were of analyticalreagent grade, and deionized water was used to prepare a mobile phase and other solutions. The five hydroxyanthraquinones (aloe-emodin, rhein, emodin, chrysophanol and physcion) were purchased from the National Institute for the Control of Pharmaceutical and Biological Products. Rhubarb (crude medicinal material) was collected from Chengdu (Sichuan Province, China). Stock solutions of each hydroxyanthraquinone were prepared as a methanol

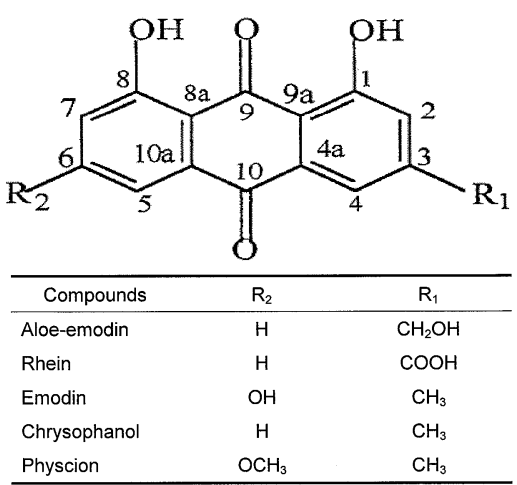

Fig. 1 Chemical structures of hydroxyanthraquinones from Rhubarb. 
Table 1 Parameters of the linearity and detection limit for the five hydroxyanthraquinones

\begin{tabular}{cclcc}
\hline Hydroxyanthraquinone & Linear range $/ \mathrm{mg} \mathrm{L}^{-1}$ & Calibration curve equation & Calibration coefficient & Detection limit/ng \\
\hline Aloe-emodin & $0.5-30$ & $C=0.006563 A-0.08525$ & 0.9999 & 0.35 \\
Rhein & $0.5-40$ & $C=0.017380 A-0.0538$ & 0.9999 & 1.12 \\
Emodin & $0.5-25$ & $C=0.008576 A-0.19423$ & 0.9999 & 0.37 \\
Chrysophanol & $0.5-30$ & $C=0.006146 A-0.05$ & 0.9999 & 0.50 \\
Physcion & $0.5-40$ & $C=0.030713 A+0.006771$ & 0.9996 & 3.13 \\
\hline
\end{tabular}

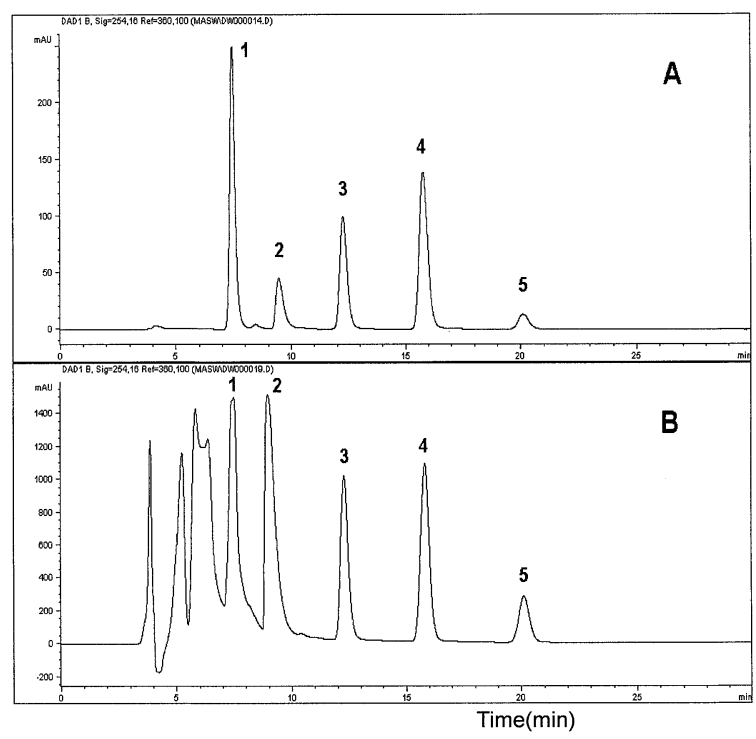

Fig. 2 HPLC chromatograms of standards (A) and rhubarb extracts (B). Compounds: 1, aloe-emodin; 2, rhein; 3, emodin; 4, chrysophanol; 5, physcion.

solution (including $0.4 \%$ dioxane) with a concentration of 100 $\mathrm{mg} / \mathrm{L}$. The standard mixtures were prepared using these stock solutions.

\section{Sample preparation}

Crushed rhubarb $(100 \mathrm{mg}$ ) was extracted with $25 \mathrm{ml}$ of ethyl acetate by placing in an ultrasonic bath for $30 \mathrm{~min}$, and was deposited for about $1 \mathrm{~h}$. A $0.5 \mathrm{ml}$ portion of this solution was transferred into a flask and evaporated to dryness by an evaporator. The residue was dissolved in $0.5 \mathrm{ml}$ of methanol. This solution was filtered through a $0.45 \mu \mathrm{m}$ membrane filter for HPLC analysis.

Freezing experimented animal samples (plasma, urine and $\mathrm{CSF}$ of rats) were thawed at $25^{\circ} \mathrm{C}$ for about $10 \mathrm{~min}$. Then, 5.0 $\mathrm{ml}$ portions of the samples were centrifuged $(3000 \mathrm{r} / \mathrm{min})$ for 20 min, and then the solutions were transferred into vials and mixed with $15 \mathrm{ml}$ of methanol-ethyl acetate (12:88, v/v) solution. After shaking for $1 \mathrm{~min}$, each sample was centrifuged (3000 r/min) for $20 \mathrm{~min}$ again and deposited for about $30 \mathrm{~min}$. The upper layer solution was transferred into a flask and evaporated to dryness. The residue was dissolved in $0.5 \mathrm{ml}$ of methanol and filtered through a $0.45 \mu \mathrm{m}$ membrane filter before HPLC analysis.

\section{HPLC conditions}

A separation column (Zorbax SB-C18, $250 \mathrm{~mm} \times 4.6 \mathrm{~mm}$ i.d. $5 \mu \mathrm{m}$, Hewlett-Packard, USA) and a guard column (Zorbax$\mathrm{C} 18,12.5 \mathrm{~mm} \times 4.6 \mathrm{~mm}$ i.d. $5 \mu \mathrm{m}$ ) were used. The mobile phase used was methanol- $0.5 \%$ acetic acid $(85: 15 \mathrm{v} / \mathrm{v})$ at a flow-rate of $0.6 \mathrm{ml} / \mathrm{min}$. The detection wavelength was set at $254 \mathrm{~nm}$. The column temperature was $30^{\circ} \mathrm{C}$, and the injection volume of samples was $20 \mu \mathrm{l}$ by a loop injector.

\section{Results and Discussion}

\section{Separation of the hydroxyanthraquinones}

The methanol content in the moble phase showed a great effect on the retention of the hydroxyanthraquinones, and with increasing the content of methanol, the hydroxyanthraquinones would be eluted more quickly. Using methanol-water $85: 15$, $\mathrm{v} / \mathrm{v})$ as the mobile phase, the analysis time was short, and the resolution was sufficient for a quantitative analysis of the five hydroxyanthraquinones. Acetic acid in the mobile phase could improve the peak tailing and the detection limits of the hydroxyanthraquinones, especially rhein. The best separation and peak shapes could be obtained when the concentration of acetic acid in the mobile phase was $0.075 \%-0.15 \%$. Therefore, methanol- $0.5 \%$ acetic acid $(85: 15 \mathrm{v} / \mathrm{v})$ was employed as the mobile phase in this work. The chromatograms of a standard mixture of the hydroxyanthraquinones and a rhubarb extract sample at the selected chromatographic conditions are shown in Fig. 2. A good separation of the five hydroxyanthraquinones was obtained.

\section{Parameters for quantitative analysis}

Linearity and detection limits. Mixtures of the five hydroxyanthraquinones with different concentrations were prepared for calibration curves. The linear range and detection limits $(S / N=$ 3 ) for the five hydroxyanthraquinones were investigated, the results are shown in Table 1. Over two orders of magnitude, a good linearity was observed for all of the analytes. The detection limits of DAD $(S / N=3)$ were between $0.35 \mathrm{ng}$ and $3.13 \mathrm{ng}$ for the hydroxyanthraquinones. The linear equation between the mass of the analytes injected and the peak area can be expressed as $C=b A+a$, where $A$ is the peak area measured by DAD, $C$ is the mass of analytes injected, and $a$ and $b$ are constants.

Dependability of the method. The recovery of the method, including all experimental procedures from the sample pretreatment to the chromatographic analysis, was measured using the rhubarb extracts and pharmacological experiment samples (plasma and urine of the experimented rats). Suitable amounts of hydroxyanthraquinones standard (aloe-emodin $25.76 \mu \mathrm{g}$, rhein $21.16 \mu \mathrm{g}$, emodin $18.68 \mu \mathrm{g}$, chrysophanol 21.56 $\mu \mathrm{g}$, physcion $18.76 \mu \mathrm{g}$ ) were added to the extracts of $100 \mathrm{mg}$ of rhubarb and $5 \mathrm{ml}$ of pharmacological experiment samples, respectively. The samples to which standards were added were pretreated and analyzed using the developed HPLC method The recoveries were expressed as the mean percentage ratio between the found amounts and the added amounts. The recoveries ranged from $83 \%$ to $103 \%$ (Table 2). As shown in Table 2, good recoveries were obtained for the rhubarb extract 


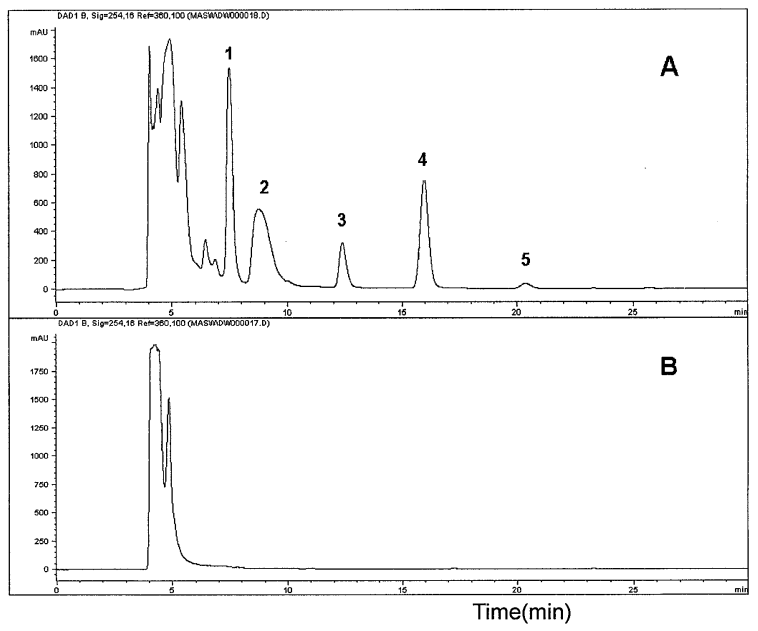

Fig. 3 HPLC chromatograms of the urine fed drug (A) and the blank urine (B). The compound numbers are the same as in Fig.2.

(96\% to $103 \%)$ and the urine of rats $(96 \%$ to $100 \%)$, but lower recoveries were obtained for the plasma of rats $(83 \%$ to $87 \%)$. It is stated that the matrix of some pharmacological samples could influence the pretreatment of the samples, and reduce the determination results of the analytes. In the sample pretreatment procedure, pure methanol, but not the mobile phase, was used for dissolving the sample residues for all samples, because we found that the recoveries of rhein were lower when the mobile phase was used for dissolving the sample residues.

The precision of the chromatographic determination for the proposed method, expressed as a relative standard deviation (RSD), was calculated by seven replicate injections of each sample (inter-day). The samples used were the same as in the recovery experiment. The RSDs of the measured concentration values of the hydroxyanthraquinones are shown in Table 3 . The RSDs of the chromatographic determination were $0.42 \%$ $5.58 \%$ for the five hydroxyanthraquinones.

\section{Analysis of samples}

The solutions of the rhubarb extracts were analyzed by the developed HPLC method under selected conditions. The peakarea calibration curves were used for a quantitative analysis of the hydroxyanthraquinones. The calculated contents of five hydroxyanthraquinones in rhubarb crude drug were $0.62 \mathrm{mg} / \mathrm{g}$ for aloe-emodin, $0.17 \mathrm{mg} / \mathrm{g}$ for rhein, $1.00 \mathrm{mg} / \mathrm{g}$ for emodin, $6.99 \mathrm{mg} / \mathrm{g}$ for chrysophanol, and $11.28 \mathrm{mg} / \mathrm{g}$ for physcion, respectively.

The developed method in this work was also applied to an analysis of the pharmacological experiment samples of rhubarb. Wistar rats (10 weeks old) were orally feed rhubarb extract (correspond to $0.5 \mathrm{~g}$ medicinal material per $100 \mathrm{~g}$ of avoirdupois of rats), and obtained the urine, plasma and CSF of the rats. The chromatograms of the urine samples of the rats sampling after and before drug-feeding are shown in Fig. 3. The determination contents of the five hydroxyanthraquinones in the plasma, urine and CSF of the rats are shown in Table 4. The contents of the drug compounds from rhubarb were much lower in the plasma and CSF of the rats, while they were over two orders of magnitude higher in the urines than in the plasma and CSF.

A simple, reliable HPLC method for the simultaneous determination of the five hydroxyanthraquinones in rhubarb and
Table 2 Recoveries for the five hydroxyanthraquinones

\begin{tabular}{lrcr}
\hline \multirow{2}{*}{ Hydroxyanthraquinone } & \multicolumn{3}{c}{ Recovery $(\%, n=7)$} \\
\cline { 2 - 4 } & Rhubarb & Plasma & Urine \\
\hline Aloe-emodin & 102.76 & 86.46 & 98.51 \\
Rhein & 96.13 & 83.42 & 95.80 \\
Emodin & 102.39 & 87.01 & 100.47 \\
Chrysophanol & 95.61 & 85.19 & 96.25 \\
Physcion & 97.43 & 82.77 & 98.27 \\
\hline
\end{tabular}

Table 3 RSDs of the measured concentration values for five hydroxyanthraquinones

\begin{tabular}{lcccc}
\hline \multirow{2}{*}{ Hydroxyanthraquinone } & \multicolumn{4}{c}{ RSD of concentration $(\%, n=7)$} \\
\cline { 2 - 5 } & Rhubarb & Plasma & CSF & Urine \\
\hline Aloe-emodin & 1.45 & 5.36 & 2.04 & 1.94 \\
Rhein & 4.95 & 1.89 & 3.09 & 0.61 \\
Emodin & 1.34 & 4.77 & 0.64 & 2.60 \\
Chrysophanol & 1.28 & 3.97 & 0.58 & 3.60 \\
Physcion & 0.42 & 5.58 & 1.44 & 0.76 \\
\hline
\end{tabular}

Table 4 Determination results of the five hydroxyanthraquinones in biological samples

\begin{tabular}{lccc}
\hline \multirow{2}{*}{ Hydroxyanthraquinone } & \multicolumn{3}{c}{ Concentration of the five anthraquinones/ } \\
$\mu \mathrm{g} \mathrm{ml}^{-1}$ \\
\cline { 2 - 4 } & Plasma (1 h) & CSF (1 h) & Urine (12 h) \\
\hline Aloe-emodin & 0.029 & 0.012 & 1.75 \\
Rhein & 0.030 & 0.073 & 5.15 \\
Emodin & 0.026 & 0.008 & 5.98 \\
Chrysophanol & 0.074 & 0.012 & 1.21 \\
Physcion & 0.018 & 0.149 & 0.56 \\
\hline
\end{tabular}

its metabolite in plasma, urine and cerebrospinal fluid (CSF) was developed. This method requires only a common stationary phase and a simple mobile phase, and gives good separation and peak shapes. This method has a high sensitivity and a wide linear range. Therefore, it is suitable for the quality control of medicinal materials and pharmacokinetic studies of rhubarb.

\section{References}

1. Y. Y. Zhou, F. Li, and L. Q. Xu, Chin. J. Pharm. Anal., 1981, 1, 263.

2. H. Oshio and N. Kawamura, Shoyakugaku Zasshi (The Japanese Journal of Pharmacognosy), 1985, 39, 131.

3. Y. Ohshima, Y. Ohno, K. Kajiyama, and K. Takahashi, J. Chromatogr. A, 1986, 360, 303.

4. Y. Kashiwada, G. Nonaka, and I. Nishioka, Chem. Pharm. Bull., 1989, 37, 999.

5. J. H. Zheng, M. Nishizawa, T. Yamakishi, I. Nishioka, H. Kaneko, H. Michibashi, and Z. C. Lou, J. Beijing Medical University, 1989, 21, 54.

6. W. Y. Luo and P. Jiang, Chin. J. Pharm. Anal., 1989, 9, 259.

7. F. L. Zhao, Y. S. Yuan, and K. W. Li, Bull. Jinling Hosp., 1999, 12, 89.

8. C. L. Liu, P. L. Zhu, and M. C. Liu, J. Chromatogr. A, 1999, $857,167$. 